\title{
POTENCIAL QUÍMICO DOS DIVERSOS MINERAIS BRASILEIROS PARA A CAPTURA DE CARBONO POR CARBONATAÇÃO MINERAL
}

\author{
G. L. A. F. ARCE ${ }^{1}$, T. G. S. NETO ${ }^{1}$, J. A. CARVALHO Jr. ${ }^{2}$, I. ÀVILA², C.M.R LUNA ${ }^{2}$, A. S. \\ SANTOS $^{1}$, V. O. FÁCIO ${ }^{2}$, J. C. SANTOS ${ }^{1}$. \\ ${ }^{1}$ Instituto Nacional de Pesquisas Espaciais, Laboratório Associado de Combustão e Propulsão \\ (INPE/LCP) \\ ${ }^{2}$ Universidade Estadual Paulista , Faculdade de Engenharia de Guaratinguetá, Departamento de \\ Energia (DEN/FEG/UNESP) \\ E-mail para contato: gretta@lcp.inpe.br
}

\begin{abstract}
RESUMO - Este trabalho avalia o potencial químico dos minerais e resíduos industriais tais como, basaltos (BAS-RS), serpentinitos (SERP-GO, SERP-MG) e escorias de aço (EAÇO-RJ) para processos de captura de carbono por carbonatação. Estas matérias primas foram escolhidas por sua abundância no território brasileiro e foram efetuadas análises para avaliar composições e comportamentos de cada mineral nas diferentes ativações térmicas e ácidas destes processos. Os resultados mostrara que maiores percentagem de metais $\mathrm{Ca}, \mathrm{Mg}$ e $\mathrm{Fe}$ foram encontradas na escória de aço (EAÇO-RJ) e nos serpentinitos (SERP-GO, SERP-MG), com aproximadamente 52, 30 e $24 \% \mathrm{p} / \mathrm{p}$ respectivamente. O SERP-GO apresentou temperatura de decomposição mais baixa $\left(700^{\circ} \mathrm{C}\right)$, sendo o mineral mais adequado para ativação térmica. Porém, o estudo de porosidade, DRX e FRX demostrou que o SERP-MG e a EAÇO-RJ são adequados para uma ativação ácida, devido à quantidade de Fe e maior área de poros, a qual favorece a extração de $\mathrm{Ca}$ e $\mathrm{Mg}$.
\end{abstract}

\section{INTRODUÇÃO}

Os níveis da concentração do dióxido de carbono $\left(\mathrm{CO}_{2}\right)$ atmosférico incrementaram-se significativamente desde a revolução industrial. Este incremento é atribuído ao $\mathrm{CO}_{2}$ antrópico gerado principalmente pelo setor energético devido à queima de combustíveis fósseis. Atualmente as concentrações atingiram $382 \pm 1$ ppm e as previsões apontam que para o ano 2100 elas atingirão os $1200 \mathrm{ppm}$, podendo prejudicar o equilíbrio do ciclo de carbono e como consequência ter algumas alterações no clima (Arce et al., 2014). Os combustíveis fósseis são uma fonte de energia importante, uma vez que sua participação na matriz energética mundial está entre $80-85 \%$. Os combustíveis fósseis têm muitas vantagens para o setor de energia, entre elas: a sua abundância, elevada densidade de energia, fácil uso, infraestrutura existente e o mais importante, seu baixo custo (Ávila, 2013). Segundo Olajire (2013) o uso deste tipo de fonte de energia nos próximos anos encara muitos desafios. No entanto, os combustíveis fósseis continuarão sendo a principal fonte de energia por um longo período, uma vez que não se vislumbra um aumento significativo na participação das fontes renováveis na matriz energética mundial (Alves et al, 2013). Embora o Brasil tenha uma matriz energética limpa, existe uma grande possibilidade que o setor de energia derivado de combustíveis fósseis ganhe espaço nos próximos anos. No entanto, o 
Brasil reconhece a necessidade de continuar tendo uma das matrizes energéticas menos poluentes do mundo para continuar com as políticas de mitigação das mudanças climáticas (Alves et al, 2013). Diante desse cenário, têm sido pesquisadas alternativas para a mitigação do $\mathrm{CO}_{2}$ na atmosfera, denominadas tecnologias de captura e sequestro de carbono (CSC) para garantir o uso contínuo dos combustíveis fósseis de forma sustentável. Muitas pesquisas apareceram, porém aquelas que visam à conversão química do $\mathrm{CO}_{2}$ num produto estável termodinâmica e ambientalmente são as que estão recebendo maior atenção. Existe a possibilidade que estes produtos sejam utilizados como matéria prima em outras indústrias (Zevenhoven \& Kavaliauskaite, 2004; Sipila et al., 2008; Bobicki et al, 2012; Fagerlund et al., 2012; Nduagu et al., 2012). Desde então aparece a carbonatação mineral como uma tecnologia de captura de carbono a qual visa mitigar o $\mathrm{CO}_{2}$ mediante sua fixação na forma de um carbonato (Zevenhovem, 2004; Olajiri, 2013).

\subsection{Carbonatação Mineral}

A carbonatação mineral é uma tecnologia baseada no processo natural de sequestro de $\mathrm{CO}_{2}$, conhecido como desagregação das rochas, as quais são conhecidas por ter um papel importante na redução histórica das concentrações de $\mathrm{CO}_{2}$ da atmosfera nos primórdios da Terra. A tecnologia de sequestro mineral, através de uma reação de carbonatação, liga o $\mathrm{CO}_{2}$ com os metais alcalinos das rochas silicatos para formar carbonatos inorgânicos sólidos. Esta reação é representada pela expressão (R1)

$$
\mathrm{MO}+\mathrm{CO}_{2} \rightarrow \mathrm{MCO}_{3}
$$

Segundo Chang et al. (2011) estes processos apresentam uma importante contribuição para as tecnologias de CSC nas proximidades das indústrias emissoras deste gás de efeito estufa, não precisando assim armazenagem deste $\mathrm{CO}_{2}$ em reservatórios geológicos. A maior vantagem da carbonatação mineral é: (a) seguridade ambiental, ou seja, os produtos são estáveis, além disso, podem ser reutilizados em outros processos industriais; e (b) uma vasta quantidade de matéria prima é encontrada no mundo inteiro (Moazzem et al., 2013). Para conseguir acelerar a taxa de reação de carbonatação mineral ao ponto que esta tecnologia se torne viável economicamente foram estudados muitos "processos de ativação". Pesquisas apontaram dois tipos de ativação: $A$ ativação química (R2), onde o uso de ácidos mediante processos de dissolução extraem metais tais como Ca, Mg e Fe em forma de sais (Wang e Maroto-Valer, 2013) e ativação térmica (R3), onde os minerais são submetidos a elevadas temperaturas a fim de degradá-los à materiais menos complexos principalmente óxidos (Balucan et al., 2013).

$(\mathrm{Mg}, \mathrm{Ca}, \mathrm{Fe}) \mathrm{SiO}_{4}+\mathrm{H}^{+} \rightarrow(\mathrm{Mg}, \mathrm{Ca}, \mathrm{Fe})^{+}+\mathrm{SiO}_{2}+\mathrm{H}_{2} \mathrm{O}$

$(\mathrm{Mg}, \mathrm{Ca}, \mathrm{Fe}) \mathrm{SiO}_{4} \rightarrow(\mathrm{Mg}, \mathrm{Ca}, \mathrm{Fe}) \mathrm{O}+\mathrm{SiO}_{2}$

Embora estes dois tipos de ativação sejam promissores para incrementar a cinética de carbonatação mineral, a escolha adequada de processos de ativação para cada matéria prima é de crucial importância para incrementar a eficiência de captura do $\mathrm{CO}_{2}$ (Chang et al., 2011). Para que este processo seja eficiente é necessário ter matérias primas com elevado conteúdo de metais como $\mathrm{Ca}, \mathrm{Mg}$ e $\mathrm{Fe}$ e baixo conteúdo de $\mathrm{Na}$ e $\mathrm{K}$. Esta característica é importante devido à que carbonatos de $\mathrm{Ca}$ e $\mathrm{Mg}$ são menos solúveis que carbonatos de $\mathrm{Na}$ e/ou $\mathrm{K}$, evitando que o $\mathrm{CO}_{2}$ retorne à atmosfera. Segundo Sipilã (2008) existem vários materiais que poderiam ser utilizados 
para a carbonatação mineral e que estão disponíveis em grandes quantidades a nível mundial. Estes materiais abrangem desde rochas silicatos (Basalto, Feldspatos, Olivinas, Piroxênios, Serpentinas, Talcos e Wollastonitas, etc.) até resíduos industriais (resíduos de cimento, resíduos de mineração, cinzas de queima de biomassa, escórias de aço, etc). Neste trabalho foram avaliadas algumas matérias primas disponíveis no Brasil para seu uso nos processos de captura de carbono por carbonatação mineral. Para isto foram avaliadas as propriedades físico-químicas de cada um das matérias primas, tais como: composição, permeabilidade e estabilidade térmica. Esta avaliação nos proporcionou um melhor entendimento dos processos de ativação que são adequados para cada matéria prima a ser utilizada.

\section{PROCEDIMENTO EXPERIMENTAL}

Foram selecionadas 4 matérias primas nacionais (3 rochas minerais e 1 resíduo industrial) para a captura de $\mathrm{CO}_{2}$ por carbonatação mineral. $\mathrm{O}$ critério de seleção das rochas minerais foi feita pela sua abundância no território nacional e o resíduo industrial foi selecionado porque o Brasil apresenta uma relevante produção de aço, sendo que esta indústria apresenta um fator de emissão de $\mathrm{CO}_{2}$ elevado ( 2 ton $\mathrm{CO}_{2}$ /ton AÇO Produzido). A Tabela 1 apresenta as matérias primas, sua origem, composição mineral, fórmula química e denominação. Cada matéria prima foi nomeada pelas primeiras letras da palavra (Basalto - BAS, Serpentinito - SERP, Escória de Aço - EAÇO) seguido do estado de origem (Rio Grande do Sul - RS, Minas Gerais - MG, Goiás - GO e Rio de Janeiro - RJ).

Tabela 1 - Matérias primas para captura de carbono por carbonatação mineral.

\begin{tabular}{|c|c|c|c|c|}
\hline $\begin{array}{c}\text { Matéria } \\
\text { Prima }\end{array}$ & Origem & $\begin{array}{l}\text { Composições } \\
\text { Minerais }\end{array}$ & $\begin{array}{c}\text { Formula Química dos } \\
\text { minerais }\end{array}$ & Nome \\
\hline \multicolumn{5}{|c|}{ Rochas Minerais } \\
\hline Basalto & $\begin{array}{l}\text { Rio Grande } \\
\text { do Sul }\end{array}$ & $\begin{array}{l}\text { Anorthite, } \\
\text { Bustamite, } \\
\text { Diopsite, } \\
\text { Enstatite, } \\
\text { Olenite, } \\
\text { Rhodonite }\end{array}$ & $\begin{array}{l}\mathrm{CaAl}_{2} \mathrm{Si}_{2} \mathrm{O}_{8} \\
\left(\mathrm{Mn}, \mathrm{Ca}_{3} \mathrm{Si}_{3} \mathrm{O}_{9}\right. \\
\mathrm{CaMgSi}_{2} \mathrm{O}_{6} \\
\mathrm{Mg}_{2} \mathrm{Si}_{2} \mathrm{O}_{6} \\
\mathrm{NaAl}_{9}\left(\mathrm{BO}_{3}\right)_{3} \mathrm{Si}_{6} \mathrm{O}_{21}(\mathrm{OH}) \\
\mathrm{Mn}^{2+}{ }_{0.9} \mathrm{Fe}^{2+}{ }_{0.02} \mathrm{Mg}_{0.02} \mathrm{Ca}_{0.05} \mathrm{SiO}_{3}\end{array}$ & BAS-RS \\
\hline Serpentinito & Goiás & $\begin{array}{l}\text { Brucite, } \\
\text { Chrysotile, } \\
\text { Lizardite, } \\
\text { Magnetite, } \\
\text { Moisanite, } \\
\text { Sodalite }\end{array}$ & $\begin{array}{l}\mathrm{Mg}(\mathrm{OH})_{2} \\
\mathrm{Mg}_{3} \mathrm{Si}_{2} \mathrm{O}_{5}(\mathrm{OH})_{4} \\
\mathrm{Mg}_{3} \mathrm{Si}_{2} \mathrm{O}_{5}(\mathrm{OH})_{4} \\
\mathrm{Fe}_{3} \mathrm{O}_{4} \\
\mathrm{SiC} \\
\mathrm{Na}_{8} \mathrm{Al}_{6} \mathrm{Si}_{6} \mathrm{O}_{24} \mathrm{Cl}_{2}\end{array}$ & SERP-GO \\
\hline Serpentinito & $\begin{array}{l}\text { Minas } \\
\text { Gerais }\end{array}$ & $\begin{array}{l}\text { Antigorite, } \\
\text { Actinolite, } \\
\text { Chrysotile, } \\
\text { akermanite, } \\
\text { Johannsenite, } \\
\text { Lizardite, } \\
\text { talc. }\end{array}$ & $\begin{array}{l}\mathrm{Mg}_{3} \mathrm{Si}_{2} \mathrm{O}_{5}(\mathrm{OH})_{4} \\
\mathrm{Ca}_{2}\left(\mathrm{Mg}_{2}, \mathrm{Fe}\right)_{5} \mathrm{Si}_{8} \mathrm{O}_{22}(\mathrm{OH})_{2} \\
\mathrm{Mg}_{3} \mathrm{Si}_{2} \mathrm{O}_{5}(\mathrm{OH})_{4} \\
\mathrm{Ca}_{2} \mathrm{MgSi}_{2} \mathrm{O}_{7} \\
\mathrm{CaMnSi}_{2} \mathrm{O}_{6} \\
\mathrm{Mg}_{3} \mathrm{Si}_{2} \mathrm{O}_{5}(\mathrm{OH})_{4} \\
\mathrm{Mg}_{3} \mathrm{Si}_{4} \mathrm{O}_{10}(\mathrm{OH})_{2}\end{array}$ & SERP-MG \\
\hline \multicolumn{5}{|c|}{ Resíduo Industrial } \\
\hline Escória de Aço & $\begin{array}{l}\text { Rio de } \\
\text { Janeiro }\end{array}$ & $\begin{array}{l}\text { Lawsoonite, } \\
\text { Larnite, } \\
\text { Ilvaite } \\
\text { Vesuvianite }\end{array}$ & $\begin{array}{l}\mathrm{CaAl}_{2} \mathrm{Si}_{2} \mathrm{O}_{7}(\mathrm{OH})_{2} \cdot\left(\mathrm{H}_{2} \mathrm{O}\right) \\
\mathrm{Ca}_{2}\left(\mathrm{SiO}_{4}\right) \\
\mathrm{CaFe}^{2+}\left(\mathrm{SiO}_{4}\right)_{2}(\mathrm{OH}) \\
\mathrm{Ca}_{10} \mathrm{Mg}_{2} \mathrm{Al}_{4}\left(\mathrm{Si}_{2} \mathrm{O}_{7}\right)_{2}\left(\mathrm{SiO}_{4}\right)_{5}(\mathrm{OH})_{4}\end{array}$ & EAÇO-RJ \\
\hline
\end{tabular}


Ambos os SERPs e o BAS foram moídos em moinho de Jarro com esferas de alumina de diversos diâmetros. A EAÇO foi moída num moinho de alta energia com esferas de aço inox.

Após a moagem da matéria prima, foi realizado o peneiramento em peneiras ASTM de 200 e 270 mesh, para atingir uma granulometria de $64 \mu \mathrm{m}$. O pó de cada matéria prima foi encaminhado para as análises de Microscopia Eletrônica de Varredura com Espectroscopia por Dispersão de Energia (MEV-EDS), Difração de Raios-X (DRX), Análises Termogravimétricos (TG/DTG) e Porosimetria de Mercúrio e Nitrogênio.

\section{RESULTADOS}

\subsection{COMPOSIÇÃO QUÍMICA}

A Figura 1 apresenta os padrões de Difração de Raios-X e na Tabela 1 apresenta-se a fórmula química de cada mineral encontrado. Como pode ser observado em todos os materiais (BAS-RS, SERP-GO, SERP-MG, e EAÇO-RJ) foram encontrados a presença de minerais com elementos químicos tais como $\mathrm{Ca}, \mathrm{Mg}$ e $\mathrm{Fe}$, adequados para a carbonatação mineral.
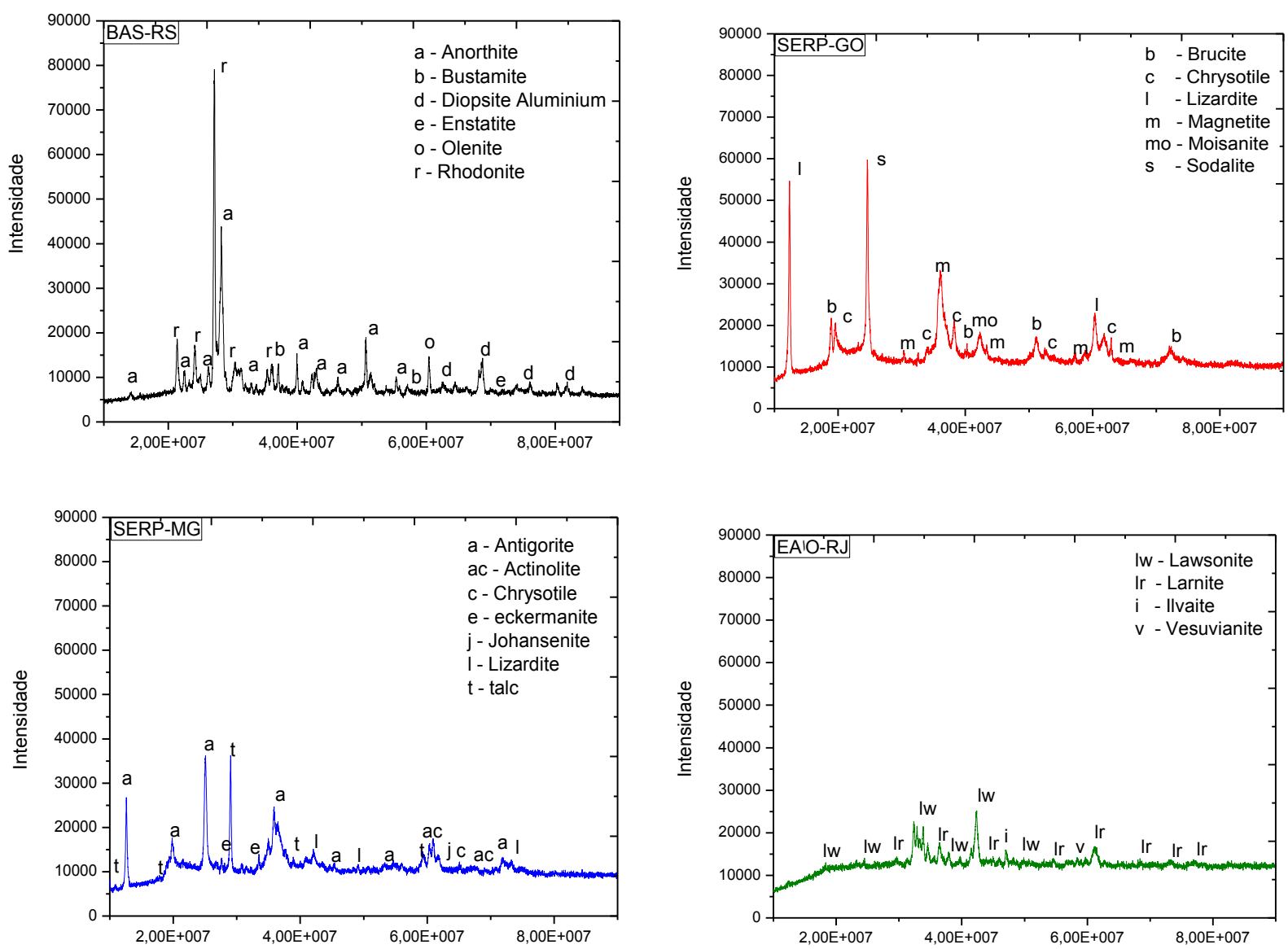

Figura 1 - Padrões de Difração de Raios-X das matérias primas. 
Segundo a Figura 1, o BAS-RS apresenta como mineral principal a anorthite $\left(\mathrm{CaAl}_{2} \mathrm{Si}_{2} \mathrm{O}_{8}\right)$ a qual é uma rocha magmática e metamórfica que apresenta teoricamente 35,84\% de $\mathrm{Al}_{2} \mathrm{O}_{3}$ (Mindata, 2014). O principal mineral no SERP-GO é o chrysotile e lizardite $\left(\mathrm{Mg}_{3} \mathrm{Si}_{2} \mathrm{O}_{5}(\mathrm{OH})_{4}\right)$ as quais são asbestos altamente serpentinizados, sendo que ambas as rochas apresentam teoricamente 46,26\% de $\mathrm{MgO}$ (Mindata, 2014), também pode ser notado a presença de brucite $\left(\mathrm{Mg}(\mathrm{OH})_{2}\right)$ e de magnetite $\left(\mathrm{Fe}_{2} \mathrm{O}_{4}\right)$, dentro de sua composição (Figura 2a). Já o principal mineral do SERP-MG é a antigorita $\left(\mathrm{Mg}_{3} \mathrm{Si}_{2} \mathrm{O}_{5}(\mathrm{OH})_{4}\right)$ com presença de actinolite $\left(\mathrm{Ca}_{2}\left(\mathrm{Mg}_{,} \mathrm{Fe}_{2}^{+}\right)_{5} \mathrm{Si}_{8} \mathrm{O}_{22}(\mathrm{OH})_{2}\right)$ a qual é uma rocha metamórfica do grupo anfíbolo e dá uma estrutura fibrosa à esta matéria prima (Figura 2b), apresenta se também o talc $\left(\mathrm{Mg}_{3} \mathrm{Si}_{4} \mathrm{O}_{10}(\mathrm{OH})_{2}\right)$. Para a EAÇO-RJ a principal mineral é a Lawsonite $\left(\mathrm{CaAl}_{2} \mathrm{Si}_{2} \mathrm{O}_{7}(\mathrm{OH})_{2} \bullet\left(\mathrm{H}_{2} \mathrm{O}\right)\right)$ e a lardite $\left(\mathrm{Ca}_{2}\left(\mathrm{SiO}_{4}\right)\right)$ com composição teórica de $16 \%$ e $66 \%$ de $\mathrm{CaO}$ (Figura 2c), respectivamente (Mindata. 2014).
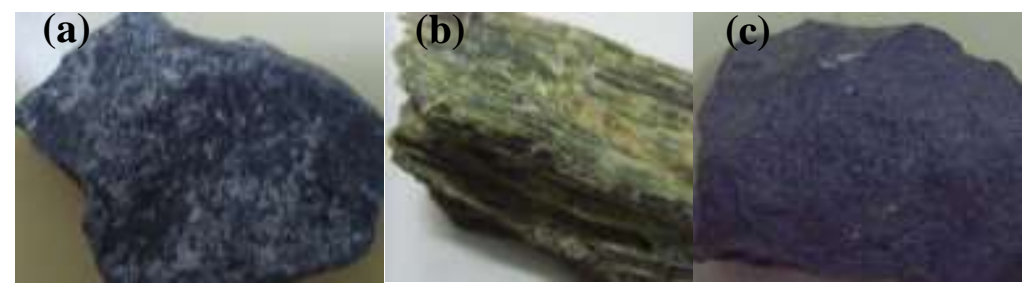

Figura 2 - Matéria prima para captura de carbono por carbonatação: (a) SERP-GO, (b) SERP-MG e (c) EAÇO-RJ.

Algumas micrografias obtidas pelo MEV das diferentes amostras são apresentadas na Figura 3. Na Figura 3a, pode ser observado que o BAS-RS apresenta alguns pontos de cor branca a qual pode ser sinal de contaminação, no entanto as estruturas não foram muito bem apreciadas. Entretanto para o SERP-GO (figura $3 b$ ) pode ser observado as diferentes estruturas do material, e para o SERP-MG (figura 3c) as estruturas fibrosas são predominantes, já no EAÇO-RJ (figura 3d) as estruturas cristalinas são mais uniformes que dos minerais silicato

$\mathrm{Na}$ Tabela 2 são apresentadas as composições químicas das matérias primas em porcentagem mássica (\%p/p) obtidas mediante a Fluorescência de Raios-X (FRX). Os resultados demonstraram que para o BAS-RS a porcentagem de $\mathrm{Si}$ é maior que as porcentagens da $\Sigma(\mathrm{Ca}, \mathrm{Mg}$ e Fe). Isto poderia dificultar a extração daqueles íons e, além disso, as porcentagens da $\Sigma(\mathrm{Ca}, \mathrm{Mg}$ e $\mathrm{Fe})$ são menores quando comparado às porcentagem de $\Sigma(\mathrm{Na}$ e K) tornando-a uma matéria prima não adequada para processos de carbonatação mineral. Entretanto, o SERP-MG, SERP-GO e a EAÇO-RJ são adequadas devido à menor quantidade de $\Sigma(\mathrm{Na} e \mathrm{~K})$, elevados conteúdos de $\Sigma(\mathrm{Ca}$, $\mathrm{Mg}$ e $\mathrm{Fe}$ ) e baixos quantidades de $\mathrm{Si}$ (isto é uma característica importante devido ao bloqueio de poros ou canais quando formado o $\mathrm{SiO}_{2} \mathrm{em}$ ambas ativações), Por último, a EAÇO-RJ seria a mais adequada devido a sua composição. Ela apresenta a mais elevada quantidade de $\Sigma(\mathrm{Ca}, \mathrm{Mg}$ e $\mathrm{Fe})$ com aproximadamente $52,59 \%$, e menor quantidade de $\mathrm{Si}(2,41 \%)$.

Pode ser observado que os serpentinitos do Brasil apresentam um baixo teor $\mathrm{Ca}$, sendo muito diferente daqueles serpentinitos encontrados na Austrália, Inglaterra e na Finlândia (Balucan et al., 2013; Wang e Maroto Valer, 2011; Teir 2008) que chegaram a ter até 1,59 \% p/p de Ca na sua composição química. 


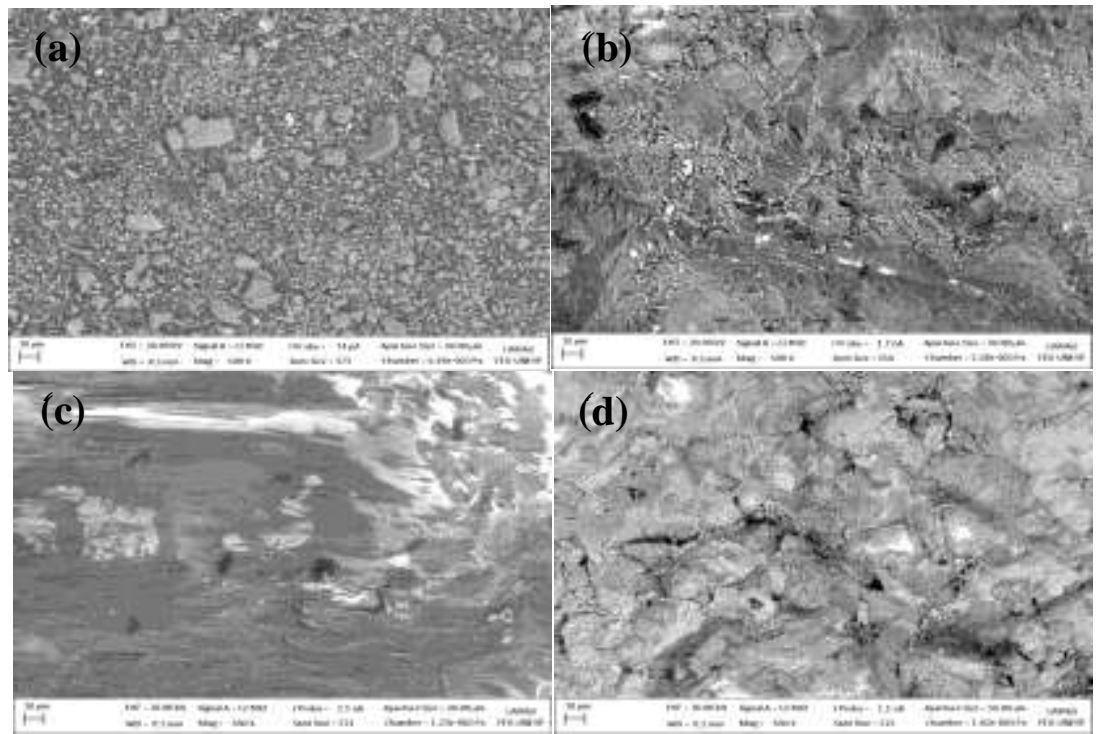

Figura 3 - MEV-EDS das matérias primas: (a) Basalto; BAS-RS (b) Serpentinito; SERP-GO

(c)Serpentinito; SERP-MG e (d)Escória de aço; EAÇO-RJ.

Tabela 2 - Composição química dos minerais em \%p/p.

\begin{tabular}{|c|c|c|c|c|}
\hline Elemento & BAS-RS & EAÇO-RJ & SERP-GO & SERP-MG \\
\hline Oxigênio $(\mathrm{O})$ & 51,33 & 39,42 & 53,75 & 51,30 \\
\hline Sódio $(\mathrm{Na})$ & 3,36 & ------ & 0,89 & 0,46 \\
\hline Magnésio $(\mathrm{Mg})$ & 1,39 & 7,43 & 20,20 & 21,78 \\
\hline Alumínio (Al) & 5,50 & 0,54 & 0,57 & 0,66 \\
\hline Silício (Si) & 22,23 & 2,41 & 13,55 & 21,12 \\
\hline Fosforo $(\mathrm{P})$ & $\begin{array}{ll}------- \\
\end{array}$ & 0,33 & ------ & \\
\hline Potássio (K) & 5,20 & ------ & 0,15 & 0,22 \\
\hline Cromo $(\mathrm{Cr})$ & & & 0,02 & 0,62 \\
\hline Cálcio $(\mathrm{Ca})$ & 1,64 & 24,71 & 0,34 & 0,16 \\
\hline Titânio (Ti) & 5,95 & ------ & 0,06 & 0,11 \\
\hline Níquel (Ni) & ------ & ------ & ------ & 0,04 \\
\hline Molibdênio (Mo) & ------- & 0,6 & 1,25 & 0,88 \\
\hline Manganês (Mn) & ------- & 4,12 & ------ & 0,03 \\
\hline Ferro $(\mathrm{Fe})$ & 3,40 & 20,45 & 9,23 & 2,60 \\
\hline Total & 100,00 & 100,00 & 100,00 & 100,00 \\
\hline \multicolumn{5}{|c|}{ Análise das Rochas segundo grupo de elementos } \\
\hline$\Sigma(\mathrm{Ca}, \mathrm{Mg}$ e $\mathrm{Fe})$ & 6,43 & 52,59 & 29,77 & 24,54 \\
\hline$\Sigma(\mathrm{Na}, \mathrm{K})$ & 8,56 & ------ & 1,04 & 0,68 \\
\hline Silício (Si) & 22,23 & 2,41 & 13,55 & 21,12 \\
\hline
\end{tabular}

\subsection{DISTRIBUIÇÃO DE TAMANHO DE PORO E PERMEABILIDADE}

As quatro amostras foram enviadas a análises de porosidade, mediante as técnicas de porosimetria de $\mathrm{Hg}$ e $\mathrm{N}_{2}$. Embora a técnica de porosimetria de $\mathrm{Hg}$ tenha grandes limitações na determinação da área superficial de poros é reconhecido que pode avaliar-se a estrutura dos poros das amostras, em relação a alguns fatores tais como a permeabilidade dos fluidos dentro das partículas (Ramli, et al., 2013). Isto é um parâmetro interessante quando se fala de ativação química. O volume de poros cumulativo (Figura 4a) está associado ao grau de permeabilidade das 
quatro amostras. Cabe destacar que este volume foi considerado até poros de $100.000 \mathrm{~A}$ de diâmetro. Os volumes obtidos acima deste valor foi considerado como volume entre partículas. Pode ser observado que o SERP-GO é a amostra menos permeável $\left(0,22 \mathrm{~cm}^{3} / \mathrm{g}\right)$, porém o SERPMG foi a mais permeável $\left(0,32 \mathrm{~cm}^{3} / \mathrm{g}\right)$ e EAÇO-RJ $\left(0,28 \mathrm{~cm}^{3} / \mathrm{g}\right)$ ficou num valor intermediário. A diferença de permeabilidade entre os serpentinitos poderia ser devido ao grau de serpentinização. Isto pode ser corroborado com o aspecto das intensidades dos picos dos difratogramas apresentados na Figura 1, os quais indicam a completa serpetinização do mineral no SERP-MG. Além do que este serpentinito tenha picos menos intensos, provavelmente indicando a presença de redes de espaços vazios na sua estrutura (Balucan et al., 2013).

A Figura $4 \mathrm{~b}$ apresenta a distribuição da estrutura porosa das matérias primas. Pode ser observada a presença de três regiões para todas as amostras. A região I representa o espaço entre as partículas, no entanto, as regiões II e III representam os espaços intra-partícula, sendo que a maior quantidade de poros encontra-se na região II com poros desde $3,15 \mu \mathrm{m}$ até $0,04 \mu \mathrm{m}(1 \mathrm{~A}=$ $0,0001 \mu \mathrm{m})$. A região III apresenta uma quantidade menor de poros para SERP-GO, SERP-MG, EAÇO-RS com poros desde $0,04 \mu \mathrm{m}$ até $0,007 \mu \mathrm{m}$. A tabela 3 apresenta os dados da estrutura de poro das matérias primas. Um dado importante é a área superficial BET encontrada com a adsorção de $\mathrm{N}_{2}$, sendo que a maior área superficial é do SERP-MG e o menor é a do SERP-GO.

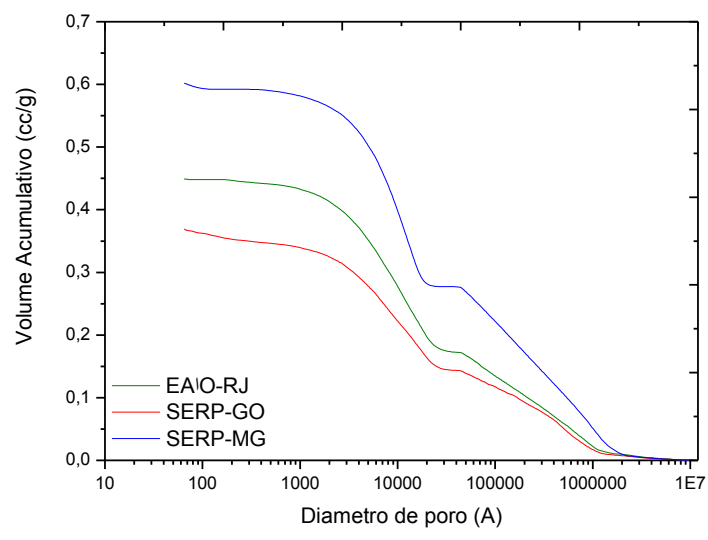

(a)

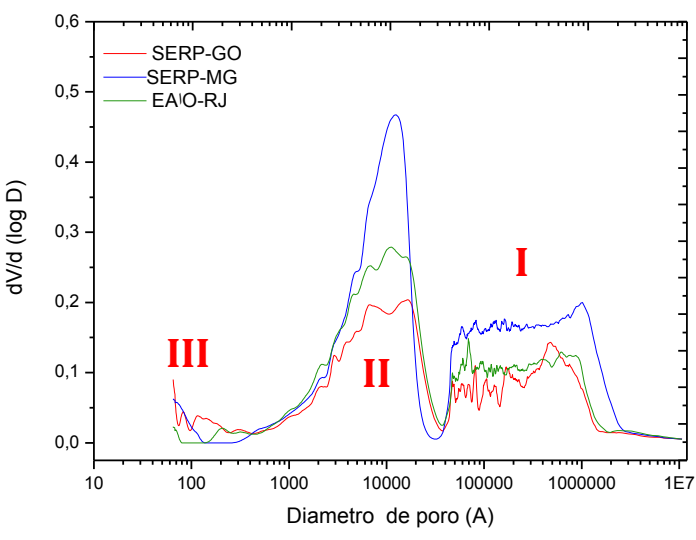

(b)

Figura 4 - Análise de porosimetria de mercúrio das matérias primas para captura de carbono por carbonatação: (a) Curva de volume de poro cumulativo (b) Curva do dif. de V por dif. do Log de diâmetro de poro.

Tabela 3 - Dados da estrutura de poro das amostras.

\begin{tabular}{|c|c|c|c|}
\hline Amostras & $\begin{array}{c}\text { Volume de Intrusão } \\
\mathrm{cm}^{3} / \mathrm{g}\end{array}$ & $\begin{array}{c}\text { Diâmetro poro médio } \\
\AA\end{array}$ & $\begin{array}{c}\text { Área Superficial BET }^{\mathrm{b}} \\
\mathrm{m}^{2} / \mathrm{g}\end{array}$ \\
\hline SERP-MG & 0,32 & 11269 & 16,9 \\
\hline SERP-GO & 0,22 & 9852 & 5,26 \\
\hline EAÇO-RJ & 0,28 & 10120 & 7,03 \\
\hline
\end{tabular}

${ }^{\mathrm{a}}$ Obtido com porosimetro de $\mathrm{Hg}$. ${ }^{\mathrm{b}}$ adsorção de $\mathrm{N}_{2}$

\subsection{PERFIL TÉRMICO DAS AMOSTRAS}

Segundo MacKenzie e McGavin (1994); Viti (2010); Balucan et al. (2013), perfis térmicos 
são de grande utilidade quando refere se a uma avaliação das matérias primas para ativações térmicas. Eles fornecem um possível mecanismo de reação e a quantidade de energia necessária para degradação da rocha até minerais menos complexos que possam ser facilmente carbonatados.

A Figura 5 apresenta as curvas TG e DTA para cada matéria prima em estudo. Um intervalo de temperaturas de $25^{\circ} \mathrm{C}$ até $1000^{\circ} \mathrm{C}$ foram aplicadas para SERP-GO, EAÇO-RJ e BAS-RS. No caso do SERP-MG foi aplicado um intervalo de temperatura desde $25^{\circ} \mathrm{C}$ até $1200^{\circ} \mathrm{C}$.
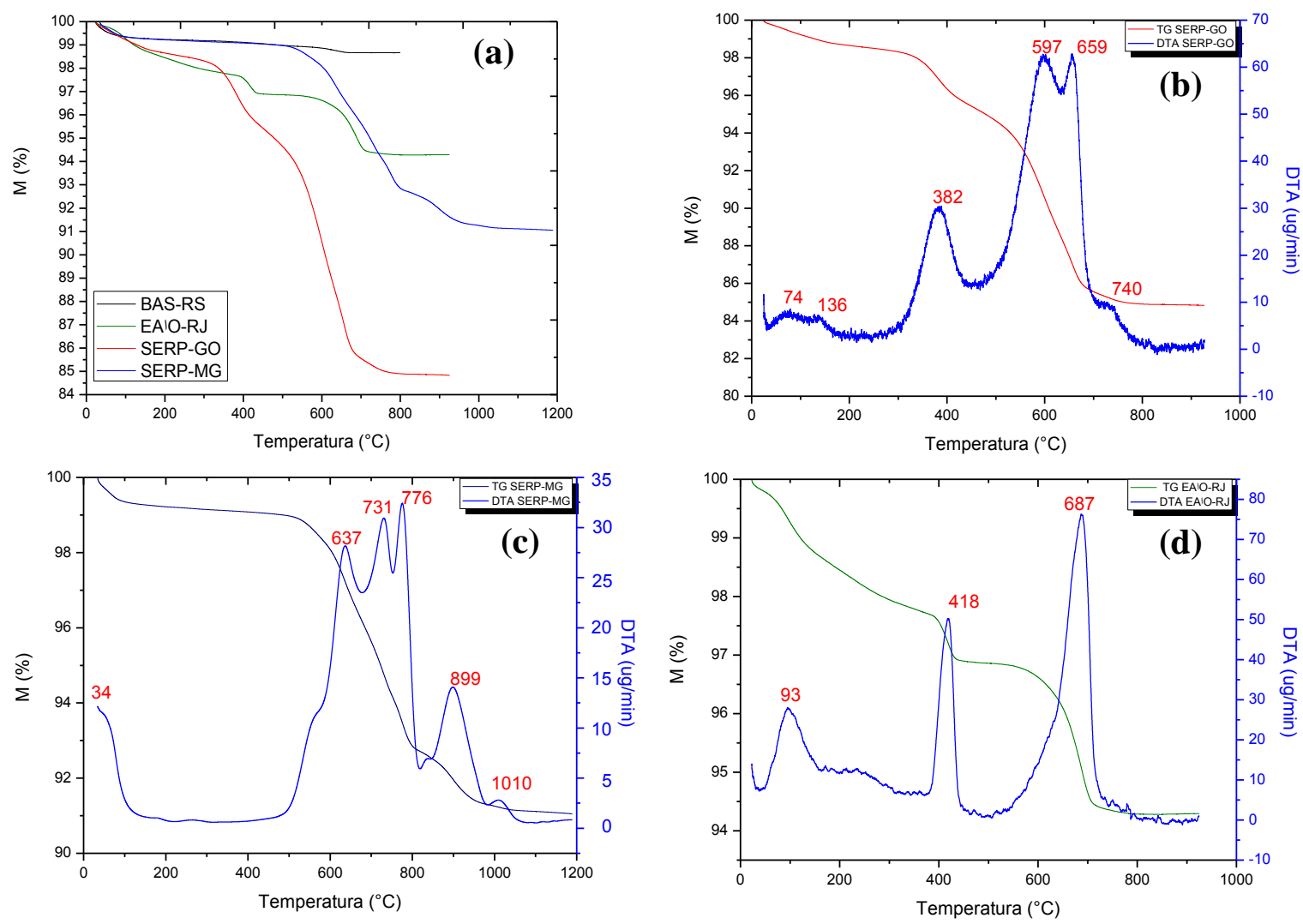

Figura 5 - Análise térmica das matérias primas. (a) Perda de massa vs temperatura, (b) TG/DTA vs temperatura do SERP_GO, (c) TG/DTA vs temperatura do SERP-MG, (d) TG/DTA vs temperaturas do EAÇO-RJ.

$\mathrm{Na}$ figura 5a exibem-se as curvas TG, onde pode ser observado que a maior perda de massa é para o SERP-GO (15,2\%), seguido do SERP-MG (8,95\%), EAÇO-RJ $(5,7 \%)$ e finalmente o BAS-RS (1,3\%). No caso da amostra de BAS-RS a estabilização na curva de perda de massa ocorreu a partir dos $700^{\circ} \mathrm{C}$. Já no caso das amostras de EAÇO-RJ e SER-GO esta estabilização ocorreu a partir dos $800^{\circ} \mathrm{C}$. E finalmente para o SERP-MG a estabilização ocorreu somente na temperatura de $1100^{\circ} \mathrm{C}$.

As figuras 5b, 5c e 5d, exibem as curvas TG/DTA do SERP-GO, SERP-MG e EAÇO-RJ respectivamente, as quais foram usadas como critério na determinação da estabilidade térmica. Pode ser notado que o BAS-RS foi retirado dessa análise devido a sua menor quantidade de metais $\mathrm{Ca}, \mathrm{Mg}$, Fe dentro na sua composição química. Todas as matérias primas em avaliação apresentam picos DTA mais intensos a partir de temperaturas maiores que $300^{\circ} \mathrm{C}$. Picos de DTA na faixa dos $100^{\circ} \mathrm{C}$ referem-se a perdas de massa por evaporação de agua (umidade). 
As figuras $5 b$ e $5 c$ apresentam os perfis térmicos de ambos os serpentinitos. Na Figura $5 b$, a curva TG exibe três perdas de massa correspondentes à vaporização d'agua, seguido da desidroxilação da "Brucite" e "Serpentinite". A curva DTA apresenta seis picos, sendo a $74^{\circ} \mathrm{C}$ atribuído à vaporização da água e a $136^{\circ} \mathrm{C}$ e $385^{\circ} \mathrm{C}$ corresponde a desidroxilação da "Brucite" amorfa e cristalina respectivamente (R4). Os mais intensos a $597-659^{\circ} \mathrm{C}$ correspondem às temperaturas de máxima desidroxilação do "Chrysotile" e "Lizardite" (R5). Por último, a 740 ${ }^{\circ} \mathrm{C}$ refere-se à formação da "Forsterite" e "Enstatite" (R6), estas avaliações térmicas são similares às encontradas na literatura (Viti, 2010). Na figura 5c, a curva TG exibe três perdas de massa correspondentes à vaporização d'agua seguido da desidroxilação do "Serpentinite" e finalmente da "Actinolite". A curva DTA apresenta sete picos, sendo os menos intensos a 34 e $265^{\circ} \mathrm{C}$ atribuídos à vaporização d'agua. Os mais intensos a 637,731 e $776^{\circ} \mathrm{C}$ referem-se às temperaturas de máxima de desidroxilação da "talc", "Antigorite", "Chrysotile" e "Lizardite" (R5), a 899 ${ }^{\circ} \mathrm{C}$ corresponde à formação de "Forsterite" e "Enstatite" (R7), e a $1010^{\circ} \mathrm{C}$ a desidroxilação de "Actinolite". Cabe ressaltar que as temperaturas de decomposição do "talc" e "actinolite" foram estimados a partir da utilização do software HSC Chemistry utilizando a reação R7 e R8.

$$
\begin{aligned}
& \mathrm{Mg}(\mathrm{OH})_{2(s)} \rightarrow \mathrm{MgO}_{(s)}+\mathrm{H}_{2} \mathrm{O}_{(g)} \\
& \mathrm{Mg}_{3} \mathrm{Si}_{2} \mathrm{O}_{5}(\mathrm{OH})_{4(s)} \rightarrow 3 \mathrm{MgO} .2 \mathrm{SiO}_{2(s)}+2 \mathrm{H}_{2} \mathrm{O}_{(g)} \\
& 3 \mathrm{MgO} .2 \mathrm{SiO}_{2(s)} \rightarrow \mathrm{Mg}_{2} \mathrm{SiO}_{4(s)}+\mathrm{MgSiO}_{3(s)}+\text { energia } \\
& \mathrm{Mg}_{3} \mathrm{Si}_{4} \mathrm{O}_{10}(\mathrm{OH})_{2(s)} \rightarrow 3 \mathrm{MgSiO}_{3(s)}+\mathrm{SiO}_{2(s)}+\mathrm{H}_{2} \mathrm{O} \\
& \mathrm{Ca}_{2} \mathrm{Mg}_{5}(\mathrm{OH})_{2} \mathrm{Si}_{8} \mathrm{O}_{22(s)} \rightarrow 2 \mathrm{CaMgSi}_{2} \mathrm{O}_{6(s)}+3 \mathrm{MgSiO}_{3(s)}+\mathrm{SiO}_{2(s)}+\mathrm{H}_{2} \mathrm{O}
\end{aligned}
$$

Comparando as curvas TG dos dois serpentinitos pode ser registrado que ambos apresentam quase o mesmo comportamento tendo três perdas de massa importantes, no entanto, a diferença encontra-se nas curvas DTA. No SERP-GO a presença de Brucite é claramente notada na curva DTA $\left(385^{\circ} \mathrm{C}\right)$, a qual é coerente com o difratograma de Raios-X. No SERP-MG não temos picos nesta temperatura na curva de DTA, porque não tem presença de Brucite dentro da composição, segundo a difração de Raios-X. Os picos mais intensos das curvas DTA (597 e $659^{\circ} \mathrm{C}$ ) para o SERP-GO estão relacionados à presença de dois tipos de serpentinitos. Neste caso, a Lizardite e Chrysotile, foram claramente exibidas, pois ambos minerais estão presentes nos padrões de DRX. No caso do SERP-MG, a presença de Antigorite, além de Lizardite e Chrysotile foi observada na curva DTA com a adição de um pico, totalizando três picos DTA intensos $\left(637,671\right.$ e $\left.779^{\circ} \mathrm{C}\right)$. A diferença entre a intensidade dos picos DTA referente à reação R6 entre SERP-MG $\left(899^{\circ} \mathrm{C}\right) \mathrm{e}$ SERP-GO $\left(740^{\circ} \mathrm{C}\right)$ pode ser devido à presença de talc e antigorite.

Já a figura 5d, apresenta a curva TG/DTA do EAÇO-RJ. NA curva TG é observado três perdas de massa a 93, 418 e $667^{\circ} \mathrm{C}$ referentes à vaporização da água, desidroxilação de Lawsoonite, Ilvaite e Vesuvianite respectivamente. Na Curva DTA o pico mais intenso a $687^{\circ} \mathrm{C}$ é devido à desidroxilação da "Ilavaite" e "Vesuvianite", já o pico menos intenso a $418^{\circ} \mathrm{C}$, atribuiuse a desidroxilação da "Lawsoonite". 


\section{CONCLUSÕES}

Neste artigo se avaliaram as propriedades físico-químicas das matérias primas do Brasil para a captura de carbono por carbonatação mineral. Dos testes realizados podemos concluir que o BAS-RS, não é uma matéria prima adequada para carbonatação mineral. Os padrões de DRX e do MEV demonstram alto grau de cristalinidade dos seus minerais e as análises de FRX mostraram que maiores quantidades de $\mathrm{Si}, \mathrm{Na}$ e $\mathrm{K}$ foram encontradas em comparação às outras matérias primas em estudo. Desta primeira etapa de análise as matérias primas adequadas foram EAÇO-RJ seguido de SERP-GO e SERP-MG.

Com as análises de porosimetria foram verificadas que a matéria prima com maior possibilidade para uma ativação química é o SERP-MG, devido á quantidade de poros do material e a sua maior permeabilidade quando comparado com as outras matérias primas.

Os resultados da termogravimetria demonstraram que o SERP-GO, EAÇO-RJ são matérias primas adequadas para ativação térmica, ambas desidroxilações ocorrem em temperaturas de 800 e $700^{\circ} \mathrm{C}$. Segundo o mecanismo de reação do SERP-GO, o calor da reação exotérmica da formação de "Forsterite" e "Enstatite" (R6) poderia ser aproveitada para um preaquecimento do mesmo material, no entanto, análises de DSC-"Differential scanning calorimetry" devem ser desenvolvidas com a finalidade de determinar a quantidade de energia necessária para tal decomposição. Isto forneceria uma idéia mais clara sob à ativação térmica.

Embora o SERP-GO estabilize em temperaturas menores, ele apresentaria um consumo de energia para desidroxilar a Brucite o que poderia ser um impedimento para o uso deste material. No entanto a ausência de Brucite no SERP-MG poderia favorecer quando se fala de requerimentos energéticos, por tanto concluímos que é necessário um estudo termoquímico de ambos os materiais.

\section{AGRADECIMENTOS}

Os autores agradecem ao projeto de pós doutorado FAPESP 2013/21244-5 e ao projeto FAPESP auxilio à pesquisa 2011/19920-7 pelo apoio à esta pesquisa. Também ao pessoal dos laboratórios do INPE/Cachoeira paulista, INPE/SJC e o laboratório de caracterização de materiais da FEG/UNESP.

\section{REFERÊNCIAS}

ALVES, N. et al. Potencial uso de serpentinito no armazenamento mineral do $\mathrm{CO}_{2}$. Quim Nova,v 36,p 773-777, 2013.

ARCE, G.L.A.F., Et al. A time series sequestration and storage model of atmospheric carbon dioxide, Ecol Model., v 272, p 24, 59-67, 2014.

ÁVILA, I. et al. The potencial evaluation of a Brazilian Serpentine as CO2 sorbent applied to the mineral carbon dioxide sequestration. In: 22nd Internacional Congress of Mechanical Enginneering, 2013, Ribeirão Preto. COBEM 2013, 2013.

BALUCAN D. R., et al. Energy cost of heat activation serpentinites for $\mathrm{CO}_{2}$ storage by mineralization. Int J Greenh Gas Control.v 17,p 225-239, 2013.

BOBICKI, et al. Carbon capture and storage using alkaline industrial wastes. Prog Energ Combust, v 38, p 302-320, 2012

CHANG EE, et al. Performance evaluation for carbonation of steel making slags in slurry reactor. $\mathrm{J}$ Hazard Mater,v 186, p 558-564, 2011. 
FAGERLUND, J., et al. CO2 fi xation using magnesium silicate minerals part 1: Process description and performance. J Energ,v 41,p 184-191,2012.

MACKENZIE KJ, MCGAVIN DDG. Thermal and mossbauer studies iron-containing hydrous silicate. Part 8 Chrysotile. Thermochim Acta. v 244, p 205-221, 1994.

MINDATA, 2014. The mineral and locality database. <http://www.mindat.org/>.Acceso em:05/06/2014.

MOAZZEN S, et al. Energy recovery opportunities from mineral carbonation process in coal fired power plant. Applied Thermal Engineering 51, pg. 281-291, 2013.

NDUAGU, E, et al. Contribution of iron to the energetics of $\mathrm{CO} 2$ sequestration in $\mathrm{Mg}$-silicates based rock. Energ Conver \& Manag, v 55, p 178-186, 2012.

OLAJIRI, A. A review of mineral carbonation technology in sequestration of $\mathrm{CO}_{2}$. J Petrol Sc Eng, v 109, p 364-392,2013.

RAMLI M, et al. Porosity, pore structure and water absorption of polymer-modified mortars: An experimental study under different curing conditions. Composites:Part B 55, pg. 221-233, 2013.

SIPILÄ, J., et al. Carbon dioxide sequestration by mineral carbonation Literature review, Åbo Akademi University Faculty of Technology Heat Engineering Laboratory, 2008.

TEIR, S., KUUSIK, R., FOGELHOLM, C., ZEVENHOVEN, R. Production of magnesium carbonates from serpentinite for long-term storage of CO2. Int J Min Proces, v 85, p 1-15,2008.

Viti, C. Serpentine minerals discrimination by thermal analysis. Am min, v 95, p 631-638,2010.

WANG, X., MAROTO-VALER, M.M.. Dissolution of serpentine using recyclable ammonium salts for $\mathrm{CO}_{2}$ mineral carbonation. Fuel, v 90, p 1229-1237, 2011.

WANG, X., \& MAROTO-VALER, M. M.. Integration of CO 2 Capture and Mineral Carbonation by Using Recyclable Ammonium Salts. ChemSusChem, v 4, p 1291-1300,2011.

ZEVENHOVEN, R., \& KAVALIAUSKAITE, I. Mineral Carbonation for Long-term CO2 Storage : an Exergy Analysis. Int J Therm, v 7(1), p 23-31,2004. 